\title{
PADRÃO DE MORTALIDADE DA COMUNIDADE JUDAICA DE BELO HORIZONTE NO SÉCULO XX
}

\section{Débora Balabram e Helenice Gobbi*}

O trabalho foi desenvolvido na Faculdade de Medicina da Universidade Federal de Minas Gerais (UFMG).

\author{
*Correspondência: \\ Faculdade de Medicina da \\ UFMG \\ Depto de Anatomia \\ Patológica e Medicina Legal \\ Avenida Alfredo Balena, \\ 190 \\ Sala 5000 \\ Belo Horizonte, MG \\ Cep: $30130-100$ \\ Tel: (31) 3248-9788 \\ Fax: (31) 3248-9664 \\ hgobbi@medicina.ufmg.br
}

\begin{abstract}
RESUMO
OBjEtivo. Fatores genéticos e ambientais são conhecidos por sua importância na gênese de grande parte das doenças. É possível estudá-los pela observação da prevalência de agravos nas populações, bem como das causas de mortalidade, buscando-se uma correlaçãa com os hábitos sociais e origens étnicas e famillares dos indivíduos. Nestes estudos, destacamse as comunidades nas quais podem-se verificar vínculos sociais e genéticos entre seus membros. A comunidade judaica de Belo Horizonte se encaixa nesses critérios. Ela se consolidou na década de 20 do século passado e, atualmente, a Federação Israelita de Minas Gerais (FISEMG) tem cadastro de aproximadamente 600 famillias. Este estudo pretende definir o padrão de mortalidade nessa comunidade, e como ele se modificou a partir de sua formação.

Métodos. Foram recuperados dados a partir dos arquivos do Instituto Histórico Israelita Mineiro, da FISEMG e dos Cemitérios Israelita e do Bonfim, oriundos de registros históricos e guias de sepultamento, no período de 1926 a 2003.

Resultados. Foram estudados 601 registros de óbitos, sendo $61,6 \%$ de homens e 38,4\% de mulheres. As doenças infectoparasitárias, dentre elas a tuberculose, ocorreram com maior freqüência nas décadas de 30 e 40 e decresceram nas décadas seguintes. As doenças do aparelho circulatório foram as causas de óbito mais prevalentes a partir da década de 40.

Conclusião. Houve mudança nos padrões de mortalidade da população judaica de Belo Horizonte.
\end{abstract}

Unitermos: Causa básica de morte. Judeus. Estatística e dados numéricos. Coeficiente de mortalidade.

\section{INTRODUÇÃO}

Fatores genéticos e ambientais são conhecidos por sua importância na gênese da maior parte das doenças. É possível estudá-los pela observação da prevalência de agravos nas populações, bem como das causas de mortalidade, buscando-se uma correlação com os hábitos sociais e origens étnicas e familiares dos indivíduos. Nestes estudos, destacam-se as comunidades com vínculos sociais e genéticos entre seus membros. A comunidade judaica de Belo Horizonte se encaixa nesses critérios. Ela se consolidou na década de 20 do século passado, com a formação da União Israelita de Belo Horizonte, apesar da presença judaica na cidade existir desde a sua formação. Atualmente, a Federação Israelita do Estado de Minas Gerais (FISEMG) tem registro de cerca de 600 famílias, sendo a maior parte delas de origem ashkenazi (judeus oriundos da Europa Oriental e da Central) $)^{1,2}$.

Este estudo pretende definir as principais causas de mortalidade na comunidade judaica de Belo Horizonte, e como estas se modificaram a partir de sua formação.

\section{Métodos}

Foram recuperados dados sobre a população judaica a partir dos arquivos do Instituto Histórico Israelita Mineiro (Rua Pernambuco, 326), da FISEMG (Rua Rio Grande do Norte, 477) e dos Cemitérios Israelita (Avenida Isabel Bueno, 1382) e do Bonfim (Rua do Bonfim, I I20), oriundos de registros históricos e de guias de sepultamento. Foram revistas certidões de óbito registradas do período de 1920 a
2003 e obtidos dados referentes a idade, gênero, nacionalidade, causa de óbito e ano de óbito. O país de nascimento, nome materno de família e alguns nomes próprios foram utilizados para identificar a origem (se ashkenazim ou sefaradim). Os sefaradim (judeus oriundos de países mediterrâneos e do norte da África) geralmente mantêm algumas características especiais em seus sobrenomes que permitem identificar sua origem ${ }^{3}$. Os dados foram coletados e analisados empregando-se o programa Epi Info versão 6.04d. Foi feita a classificação da causa básica de morte utilizando os padrões adotados pelo CID- IO (Classificação Internacional de Doenças - versão I0), segundo recomendação do Ministério da Saúde 4 .

Os dados relativos às causas de morte foram trabalhados de modo a obter-se a mortalidade proporcional ao longo das décadas.

\section{Resultados}

Foram obtidos e analisados 60 I registros de óbitos de indivíduos da comunidade judaica, sendo $61,6 \%$ de homens e 38,4\% de mulheres. O primeiro registro data de 02/03/1926 e o último de 01/06/2003.

Quanto à origem dos indivíduos, obtivemos um total de 400 $(66,6 \%)$ ashkenazim (judeus vindos da Europa Oriental) e 39 (6,5\%) sefaradim (judeus vindos, em sua maioria, de países árabes), sendo o restante 162 indivíduos (27\%) de origem indeterminada.

\section{I) Distribuição das causas de morte ao longo das décadas}

Observou-se que as doenças do aparelho circulatório foram as principais causas de morte na comunidade judaica a partir da década de 
Figura I - Distribuição da mortalidade proporcional na comunidade judaica de Belo Horizonte ao longo das décadas
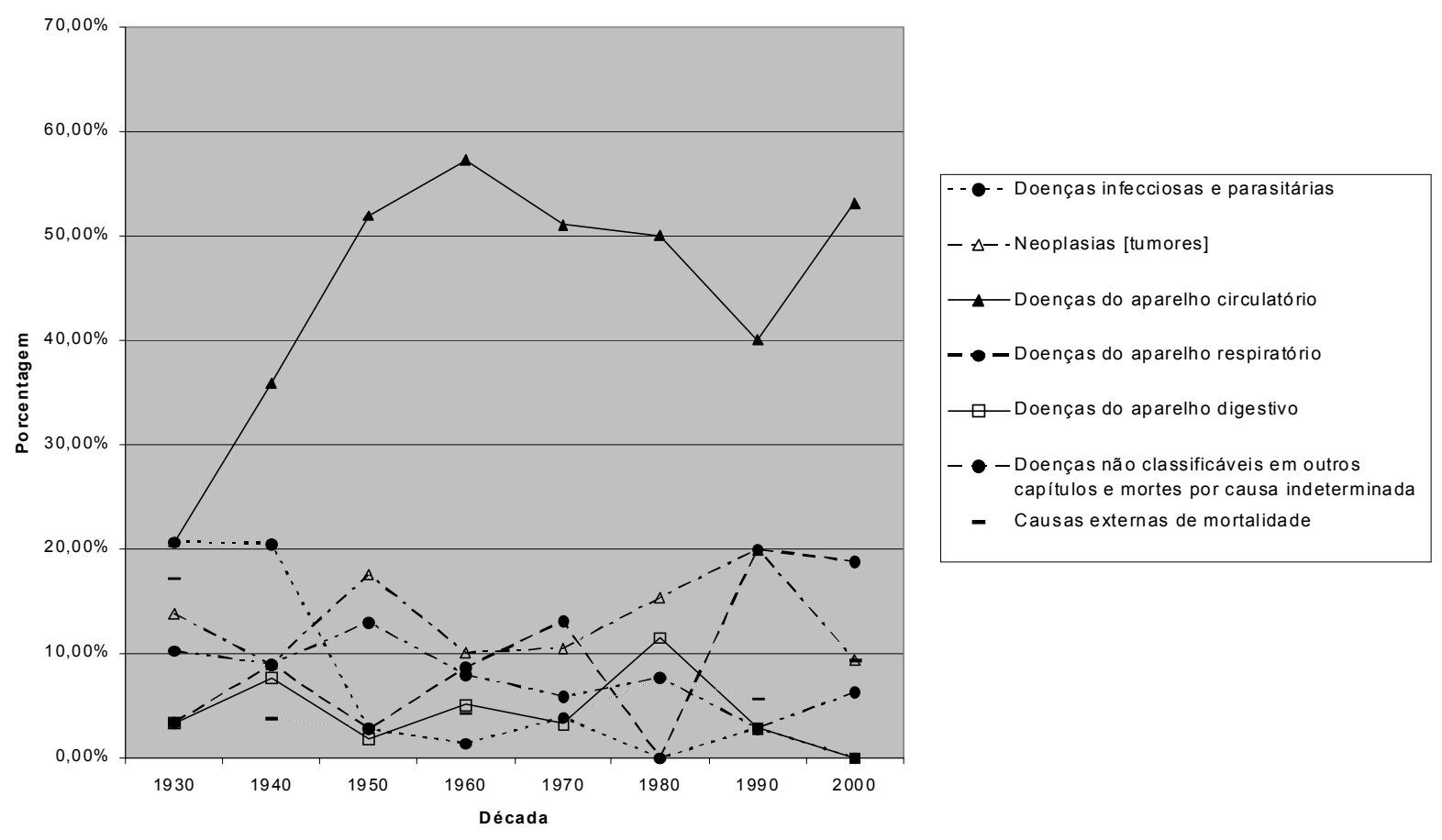

40 (Figura I). Elas causaram 292 óbitos (48,6\%). As doenças isquêmicas do coração foram as mais prevalentes, causando 93 (31,9\%) óbitos, seguidas pela insuficiência cardíaca, com 64 casos $(21,9 \%)$ e pelas doenças cerebrovasculares, com 58 casos (19,9\%).

Nas décadas de 30 e 40, as doenças infecciosas também se destacaram, com ocorrência semelhante à das doenças do aparelho circulatório na década de 30. A principal doença infecciosa relacionada a óbito foi a tuberculose, responsável por 24 óbitos (68,6\% do total de mortes por doenças infecciosas em todas as décadas). A partir da década de 50, houve uma diminuição progressiva do número de óbitos por doenças infecciosas, sendo ultrapassadas por doenças do aparelho cardiovascular, respiratório, neoplasias e outras.

O aparelho respiratório foi responsável por 56 óbitos (9,3\%). A pneumonia foi a principal doença causadora de óbito neste grupo, tendo sido responsável por 18 óbitos (32,1\%). Foram encontradas 17 mortes por edema agudo pulmonar sem outras especificações $(30,4 \%)$.

As causas externas foram responsáveis por 30 óbitos (0,5\%). A maior parte delas se deveu a acidentes, não tendo sido observada uma taxa maior nas décadas de 30 e 40, quando havia perseguições aos judeus em diversas partes do mundo.

As neoplasias malignas causaram 72 mortes (12\%). Em 18 óbitos por câncer (25\%), o sítio primário da neoplasia não foi especificado nos atestados, constando apenas termos como "caquexia neoplásica", "carcinomatose", etc. A Tabela I mostra as freqüências relativas encontradas para cada tumor. O sítio mais freqüente de neoplasia foi o tubo
Tabela I - Mortalidade proporcional por neoplasias malignas como causa de óbito na população judaica de Belo Horizonte no período de 02/03/1936 a 01/06/2003

\begin{tabular}{|c|c|c|}
\hline Neoplasia & Freqüência absoluta (n) & Freqüência relativa (\%) \\
\hline Tubo digestivo & 17 & $2,90 \%$ \\
\hline Mama e ovário & 6 & $1,00 \%$ \\
\hline Fígado & 5 & $0,83 \%$ \\
\hline Pulmão & 5 & $0,83 \%$ \\
\hline Sistema hematopoético & 4 & $0,66 \%$ \\
\hline Outros & 17 & $2,83 \%$ \\
\hline Sítio não especificado & 18 & $3,00 \%$ \\
\hline
\end{tabular}

digestivo (29 casos, 40,3\%). Foram encontrados cinco casos de câncer de mama (6,9\%) e um caso de tumor de ovário (I,4\%). Havia também cinco casos de tumores de fígado, e apenas um caso de morte por câncer do colo do útero. Dentre os tumores do tubo digestivo, os principais foram o de estômago, com nove casos (I2,5\% do total), e colorretais, com oito casos $(11,1 \%)$.

Um total de 49 óbitos $(8,2 \%)$ não pode ser classificado em categorias adequadas de mortalidade do CID- I0, entrando no capítulo relativo a "achados não classificados em outra parte". Dentre eles, constaram termos como "senilidade", "caquexia", etc. Este capítulo também inclui os óbitos por causa indeterminada, que foram, ao todo, $17 \operatorname{casos}(2,8 \% \text { do total })^{4}$. 
Figura 2 - Mudanças na idade média ao óbito dos membros da comunidade judaica de Belo Horizonte ao longo das décadas

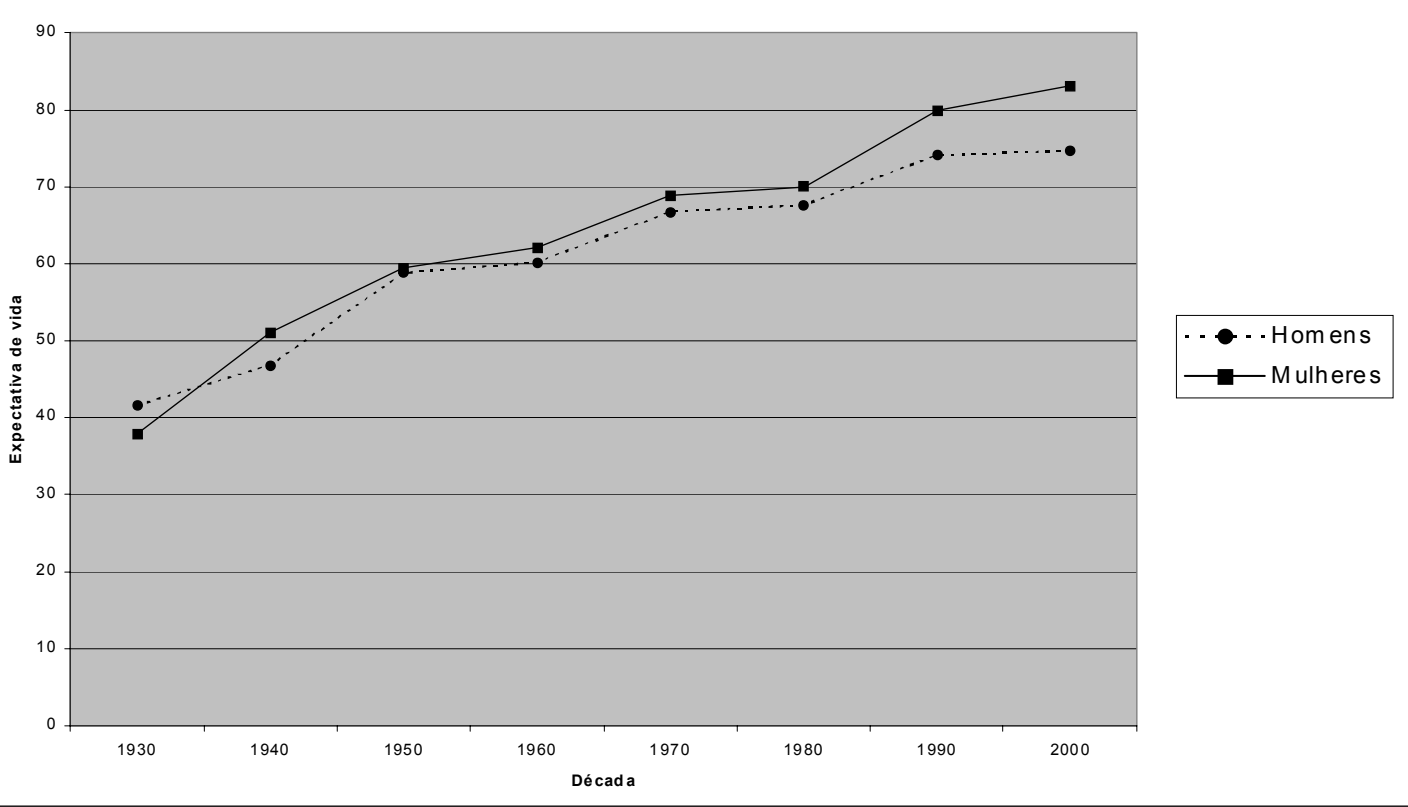

\section{2) Aumento da idade média ao óbito ao longo das décadas}

A partir da década de 30 , foi observado um aumento contínuo da idade média ao óbito dentre os membros da comunidade (Figura 2), passando de 40 anos, na década de 30, para 77 anos, na década de 90. Observou-se também que a idade média ao óbito das mulheres foi constantemente maior que a dos homens a partir da década de 40.

\section{Discussão}

Neste trabalho, avaliamos as causas de morte dentre os membros da comunidade judaica de Belo Horizonte no período de 1926 a 2003. Observamos mudanças significativas entre as causas de óbito e aumento da idade média ao óbito durante o período analisado.

Tuberculose foi a principal causa de óbito por doença infecciosa nas décadas de 1930, 1940 e 1950, reduzindo sensivelmente sua mortalidade proporcional a partir da década de 1960. As mortes decorrentes de tuberculose a partir de então devem-se a seqüelas da doença. É bem provável que esta redução tenha ocorrido pela intensificação da atuação do Estado no controle da tuberculose, que culminou com a Campanha Nacional Contra a Tuberculose, em 1946, juntamente com o advento dos quimioterápicos contra a doença, na mesma década 5 .

Observamos aumento das mortes por neoplasia a partir de 1950. o mesmo ocorrendo com as mortes por doenças cardiovasculares.

A freqüência encontrada para registro de óbito por tumores de fígado como causa de morte foi maior que a esperada para a população. A incidência média de hepatocarcinoma no mundo é de 1/100.000 indivíduos 6 . É provável que as mortes registradas como "tumores hepáticos" representem neoplasias metastáticas para o fígado, que são mais freqüentes, e não tumores primários do fígado, como mostra a literatura ${ }^{6}$.
Considerando tratar-se de uma comunidade judaica de maioria ashkenazi, era de se esperar uma incidência maior de mortes por câncer de mama e ovário, não verificada no presente trabalho. Tem sido descrita maior incidência de mutações nos genes BRCA I e BRCA 2 nestas populações, que são consideradas fatores de risco para o surgimento do câncer de mama e de ovário familiares $7,8,9,10,11$. Também seria esperado encontrar maior número de carcinomas de mama familiares e hereditários, com suas respectivas características. No entanto, neste trabalho foram encontrados apenas cinco casos de óbito por câncer de mama (0,8\% em relação ao total e 6,9\% em relação às neoplasias malignas) e um por câncer de ovário $(0,2 \%$ em relação ao total e I,4\% em relação às neoplasias malignas). Não havia relação de parentesco identificável pelos nomes de família dentre os registros de óbito por câncer de mama nesta casuística. As idades dos indivíduos afetados por câncer de mama e ovário variaram de 46 a 84 anos. Não houve casos de câncer de mama entre homens. É possível que alguns casos de câncer de mama possam ter sido incluídos dentre os cânceres que entraram na categoria indeterminado (por exemplo, categorizados como "caquexia neoplásica", "carcinomatose"). Apenas cinco dos 18 casos relatados como "indeterminados" ocorreram entre mulheres. Um estudo brasileiro avaliou a incidência de mortes por câncer de mama nas comunidades judaicas de Porto Alegre e do Rio de Janeiro, e concluiu não haver diferenças em relação à população geral destas localidades no período estudado ${ }^{3}$. Os autores admitem que, dentre os fatores responsáveis pela incidência semelhante, poderiam ser incluídos a alimentação e outros hábitos.

Houve baixa mortalidade por câncer de colo de útero na comunidade judaica de Belo Horizonte, semelhante à observada em mulheres judias de outros países. Uma das leis entre os judeus exige que todos os homens sejam circuncisados uma semana após o nascimento ${ }^{12}$. A circuncisão permite 
melhor higienização da glande peniana, diminuindo o acúmulo dos vírus HPV, que têm sido associados à patogênese do câncer de colo de útero e pênis, e portanto diminui a transmissão do vírus às mulheres ${ }^{13}$.

As fontes documentais do presente estudo têm limitações, em especial em relação à qualidade do preenchimento das causas de morte nos atestados de óbito. Fatos semelhantes são relatados por outros autores que consideram que o preenchimento inadequado dos atestados de óbito é uma limitação à sua utilização como fonte de dados confiável ${ }^{14}$. Houve diversos casos em que a causa de morte descrita foi "colapso"; estes casos foram incluídos na categoria "doenças não classificáveis em outros capítulos e mortes por causa indeterminada".

As mortes por doenças do aparelho respiratório podem ter sido superestimadas, já que os casos descritos apenas como "edema agudo de pulmão" foram incluídos nesta categoria, e é provável que na realidade eles se devessem a doença cardíaca descompensada, que pode desencadear o edema. Foram encontradas 17 mortes por edema agudo pulmonar, que respondem por um total de 30,4\% das mortes por doenças do aparelho respiratório. Dentre as mortes por neoplasia, como citado anteriormente, também houve dificuldade em se determinar o sítio primário ou tipo de neoplasia pelo fato do atestado de óbito conter termos genéricos como "caquexia neoplásica" ou "carcinomatose".

\section{ConClusão}

Os dados deste trabalho permitem concluir que mudanças claras foram observadas nas causas de mortalidade da comunidade judaica de Belo Horizonte ao longo das décadas. Em especial, notou-se uma substituição das mortes por doenças infecto-parasitárias, mais prevalentes nas décadas de 30 e 40, pelas mortes por doenças degenerativas e cardiovasculares, que aumentaram progressivamente a partir da década de 30. Percebeu-se uma variação na freqüência das neoplasias com baixa ocorrência de óbito devido a câncer de mama e de colo uterino. Observou-se aumento contínuo da idade média ao óbito ao longo das décadas.

\section{Conflito de interesse: não há.}

\section{Agradecimento}

Agradecemos a Moysés Balabram e às inúmeras pessoas que contribuíram para a realização deste trabalho.

\section{SUMMARY}

\section{Mortality Pattern of Jewish Community of Belo Horizonte in the XX $\mathbf{X}^{\mathrm{Th}}$ Century}

OBETIVE. Genetic and environmental factors are known for their importance in the genesis of the majority of diseases. It is possible to study them through the observation of prevalence of diseases, and also the mortality causes, relating it to social habits and ethnical and familial origins of the individuals. In those studies, communities in which there are social and genetic links stand out. The jewish community of Belo Horizonte fits these criteria. It has been consolidated in the $20^{\text {th }}$ decade of the last century. Nowadays the Federação Israelita de Minas Gerais (FISEMG) has records of approximately 600 families. The purpose of this study is to define the mortality pattern in this community, and how it has changed since the community'sformation.

METHODS. Data was recovered from the files of the Instituto Histórico Israelita Mineiro, the FISEMG and from the cemeteries Israelita and Bonfim.

RESULTS. 601 death registries have been studied, $61,6 \%$ belonging to men and $38,4 \%$ to women. The diseases of the circulatory system were the most frequent cause of death since the decade of 40 , and infectious diseases, including tuberculosis, deserve note in the decades of 30 and 40.

CONCLUSION. There was a change in death pattern of the jewish community of Belo Horizonte. [Rev Assoc Med Bras 2006; 52(6): 409-12]

KEY WORDS: Underlying cause of death. Jews. Statistics and numerical Data. Mortality coefficient.

\section{REFERÊNCIAS}

I. Cuperschmid EM. Judeus entre dois mundos: A formação da comunidade judaica de Belo Horizonte; 1922- 196 I [tese]. Belo Horizonte: Faculdade de Filosofia e Ciências Humanas, Universidade Federal de Minas Gerais; 1977.

2. Pfeffer RS. A comunidade judaica de Belo Horizonte: formação de uma identidade étnica particular numa sociedade diferenciada e plural [dissertação]. Belo Horizonte: Faculdade de Filosofia e Ciências Humanas, Universidade Federal de Minas Gerais; 1993.

3. Koifman S, Koifman JR. Breast cancer mortality among Ashkenazi Jewish women in Sao Paulo and Porto Alegre, Brazil. Breast Cancer Res. $2001 ; 3: 270-5$.

4. Organização Mundial de Saúde. Classificação Internacional das Doenças, versão 10 (CID I0). Tradução do Centro Colaborador da OMS para a Classificação de Doenças em Português. $6^{a}$ ed. São Paulo: Editora da Universidade de São Paulo; 2001 .

5. Fernandes TMD, Almeida ABS, Nascimento DR. Introdução. In: Memória da Tuberculose. Disponível em: http://www.coc.fiocruz.br/tuberculose/ introducao.htm [citado 2 I ago 2004]

6. Doherty GM, Way LW. Liver \& portal venous system. In: Doherty GM, Way LW, editors. Current surgical diagnosis and treatment. I I th $\mathrm{ed}$. New York: Lange Medical Books/McGraw-Hill; 2003. p.57I-94.

7. Greene, MH. Genetics of breast cancer. Mayo Clin Proc. 1997;72:54-65.

8. Goldgar DE, Reilly PR. A common BRCAI mutation in the Ashkenazim. Nat Genet. 1995; I I: | 13-4.

9. Struewing JP, Abeliovich D, Peretz T, Avishai N, Kaback MM, Collins FS, et al. The carrier frequency of the BRCAI I85delAG mutation is approximately I percent in Ashkenazi Jewish individuals. Nat Genet. | 995; | | : | 98-200.

10. Neuhausen S, Gilewski T, Norton L, Tran T, Macguire P, Swensen J, et al. Recurrent BRCA2 6174delT mutations in Ashkenazi Jewish women affected by breast cancer. Nat Genet. 1996; 13:126-8

I I. Foulkes WD, Wong N, Brunet JS, Begin LR, Zhang JC, Martinez JJ, et al. Germ-line BRCAI mutation is an adverse prognostic factor in Ashkenazi Jewish women with breast cancer. Clin Cancer Res. 1997; 3(12 PtI):2465-9

12. Donin $H$ H. O ser judeu: guia para a observância judaica na vida contemporânea. São Paulo: Editora Sêfer; 1985. p.288-96: nascimento.

13. Castellsaguè X, Bosch FX, Muñoz N, Meijer CJLM, Shah KV, Sanjosé S, et al. Male circumcision, penile human papillomavirus infection, and cervical câncer in female patients. N Engl J Med. 2002;346: I 005- | | | 2.

14. Conselho Federal de Medicina. $\bigcirc$ atestado de óbito e a formulação das políticas públicas. J Med. 2004/2005; I 52:22. Aceito para publicação: 01/08/2006 